\title{
POSITIVITY CONDITIONS FOR BIHOMOGENEOUS POLYNOMIALS
}

\author{
David W. Catlin and John P. D'Angelo
}

\section{Introduction}

In this paper we continue our study of a complex variables version of Hilbert's seventeenth problem by generalizing some of the results from [CD]. Given a bihomogeneous polynomial $f$ of several complex variables that is positive away from the origin, we proved that there is an integer $d$ so that $\|z\|^{2 d} f(z, \bar{z})$ is the squared norm of a holomorphic mapping. Thus, although $f$ may not itself be a squared norm, it must be the quotient of squared norms of holomorphic homogeneous polynomial mappings. The proof required some operator theory on the unit ball. In the present paper we prove that we can replace the squared Euclidean norm by squared norms arising from an orthonormal basis for the space of homogeneous polynomials on any bounded circled pseudoconvex domain of finite type. To do so we prove a compactness result for an integral operator on such domains related to the Bergman kernel function. Recall that the Bergman kernel function $B$ for a domain $\Omega$ is the integral kernel for the operator $P$ that projects $L^{2}(\Omega)$ to the closed subspace $A^{2}(\Omega)$ of holomorphic functions in $L^{2}(\Omega)$.

We prove the following results.

Proposition 1. Suppose that $\Omega$ is a bounded pseudoconvex domain in $\mathbb{C}^{n}$ for which the $\bar{\partial}$-Neumann operator $N$ is compact. Let $M$ be a pseudodifferential operator of order 0 . Then the commutator $[P, M]$ is compact on $L^{2}(\Omega)$.

Theorem 1. Suppose that $\Omega$ is a smoothly bounded pseudoconvex domain of finite type in $\mathbb{C}^{n}$, with Bergman kernel $B(z, \bar{\zeta})$. Let $g$ be a smooth function on $\bar{\Omega} \times \bar{\Omega}$ that vanishes on the boundary diagonal. Then the operator on $L^{2}(\Omega)$ with integral kernel $g B$ is compact.

Theorem 2. Suppose that $\Omega$ is a smoothly bounded pseudoconvex circled domain in $\mathbb{C}^{n}$ of finite type. For each integer $d$, let $\Phi^{d}=\left(\Phi_{1}^{d}, \ldots, \Phi_{N}^{d}\right)$ denote an orthonormal basis for the homogeneous polynomials of degree $d$ on $\Omega$. Let $f$ be a bihomogeneous polynomial that is positive away from the origin. Then

Received January 31, 1997.

The frist author is supported by NSF grant DMS-94015480.

The second author is supported by NSF grant DMS-9304580 at IAS and by MSRI. 
there is an integer $d_{0}$ (depending on $f$ ) such that, for each $d \geq d_{0}$, there is a homogeneous polynomial mapping $h$ such that

$$
\left\|\Phi^{d}(z)\right\|^{2} f(z, \bar{z})=\|h(z)\|^{2} .
$$

In section III we interpret Theorem 2 and the theorem from [CD] in terms of Hermitian line bundles over complex projective space. In a future paper we will prove a related differential geometric theorem involving metrics on Hermitian line bundles over compact complex manifolds.

The second author acknowledges useful discussions with Yum-Tong Siu, Steve Bradlow, and Alex Tumanov. He also acknowledges support from MSRI. The first author acknowledges support from NSF. The authors acknowledge helpful comments by the referee.

\section{Needed facts about compact operators}

In this section we write $(u, v)$ for the inner product on a Hilbert space, and $\|u\|^{2}$ for the squared norm. An operator $A: H_{1} \rightarrow H_{2}$ between Hilbert spaces is compact if whenever $f_{j}$ is a bounded sequence in $H_{1}$, then $A\left(f_{j}\right)$ has a convergent subsequence in $\mathrm{H}_{2}$. We convert this into an estimate:

Lemma 1. Suppose that $A: H_{1} \rightarrow H_{2}$ is an operator between Hilbert spaces. Then $A$ is compact if and only if, for all positive $\epsilon$, there is a compact operator $B=B_{\epsilon}$ and a positive constant $C_{\epsilon}$ such that the following estimate holds:

$$
\|A f\|^{2} \leq \epsilon\|f\|^{2}+C_{\epsilon}\left\|B_{\epsilon} f\right\|^{2} .
$$

Proof. If $A$ is compact, we may take $B=A$ and $C_{\epsilon}=1$. Conversely, we suppose that (1) holds. Let $f_{j}$ be a bounded sequence in the domain; we will construct a subsequence whose image under $A$ is Cauchy. Consider a positive integer $n$. From (1) we have

$$
\left\|A\left(f_{j}\right)-A\left(f_{k}\right)\right\|^{2} \leq \epsilon\left\|f_{j}-f_{k}\right\|^{2}+C_{\epsilon}\left\|B_{\epsilon}\left(f_{j}-f_{k}\right)\right\|^{2}
$$

Since $\left\|f_{j}\right\|$ are bounded, the first term can be made smaller than $\frac{1}{2 n}$ by choosing $\epsilon$ small enough. The second can then be made smaller than $\frac{1}{2 n}$ by extracting a subsequence (still labeled the same) for which $B_{\epsilon} f_{j}$ converges and then choosing $j$ and $k$ sufficiently large. Thus for each $n$ there is a subsequence such that $\left\|A\left(f_{j(n)}\right)-A\left(f_{k(n)}\right)\right\|^{2}<\frac{1}{n}$. Extracting the diagonal subsequence gives a subsequence whose image under $A$ is Cauchy. Therefore $A$ is compact.

In order to prove (1) in specific cases we will use the standard remark that, given $\epsilon>0$, there is a positive constant $C_{\epsilon}$ so that

$$
|(u, v)| \leq \epsilon\|u\|^{2}+C_{\epsilon}\|v\|^{2} .
$$


We sometimes write $s c$ for $\epsilon$ and $l c$ for $C_{\epsilon}$.

Let $\Omega$ be a smoothly bounded pseudoconvex domain in $\mathbb{C}^{n}$. We assume that the reader is familar with the $\bar{\partial}$-Neumann problem. See $[\mathrm{C} 2, \mathrm{D}, \mathrm{FK}, \mathrm{K}]$ for example. We have the Hodge decomposition on $(0, q)$ forms given by $I=$ $\left(\bar{\partial}^{*} \bar{\partial}+\overline{\partial \partial}^{*}\right) N+H$ where $H$ is the harmonic projector. For pseudoconvex domains in $\mathbb{C}^{n}$, the operator $H$ on $(0, q)$ forms vanishes except when $q=0$, in which case it equals the Bergman projection $P$. Thus on $(0, q)$ forms for $q \geq 1$, the $\bar{\partial}$-Neumann operator $N$ satisfies $\left(\bar{\partial}^{*} \bar{\partial}+\overline{\partial \partial}^{*}\right) N=I$.

The Bergman kernel function $B(z, \bar{w})$ for a bounded domain $\Omega$ is the integral kernel of the operator $P$ that projects $L^{2}(\Omega)$ onto the closed subspace $A^{2}(\Omega)$ of holomorphic functions in $L^{2}(\Omega)$. Suppose that the collection $\left\{\phi_{\alpha}\right\}$ forms a complete orthonormal set for $A^{2}(\Omega)$. Then the Bergman kernel satisfies

$$
B(z, \bar{\zeta})=\sum \phi_{\alpha}(z) \overline{\phi_{\alpha}(\zeta)}
$$

Kohn's formula relates the $N$ operator to the Bergman projection $P$; it states that $P=I-\bar{\partial}^{*} N \bar{\partial}$. Here the $N$ operator is defined on $(0,1)$ forms. The image of $N$ is contained in the domain of $\bar{\partial}^{*}$. For forms of all degrees this remains true; then $\bar{\partial}^{*} N$ maps $(0, q)$ forms in $L^{2}(\Omega)$ to $(0, q-1)$ forms in $L^{2}$, and its adjoint $N \bar{\partial}$ maps $(0, q-1)$ forms in $L^{2}$ to $(0, q)$ forms in $L^{2}$.

We recall (See $[\mathrm{D}]$ ) that $\Omega$ is of finite type if there is a bound on the order of contact of ambient complex analytic varieties with $\mathrm{b} \Omega$ at all points. Domains of finite type satisfy subelliptic estimates [C1], and hence the $\bar{\partial}$-Neumann operator is compact. Another consequence [Ke] is that the Bergman kernel function is smooth on $\bar{\Omega} \times \bar{\Omega}$ away from the boundary diagonal. See [HI] for recent results concerning compactness of the $\bar{\partial}$-Neumann operator.

Lemma 2. Suppose that the $\bar{\partial}-N e u m a n n$ operator $N$ is compact on the spaces of forms of type $(0, q)$ and $(0, q+1)$ in $L^{2}(\Omega)$. Then $\bar{\partial}^{*} N$ and $N \bar{\partial}$ are compact.

Proof. Since $N \bar{\partial}$ on $(0, q)$ forms is the adjoint of $\bar{\partial}^{*} N$ on $(0, q+1)$ forms, it suffices to prove that $\bar{\partial}^{*} N$ is compact. Using the Hodge decomposition formula $I=\left(\bar{\partial}^{*} \bar{\partial}+\overline{\partial \partial}^{*}\right) N+H$ we see that

$$
\left\|\bar{\partial}^{*} N f\right\|^{2}=\left(\overline{\partial \partial}^{*} N f, N f\right)=(f, N f)-\left(\bar{\partial}^{*} \bar{\partial} N f, N f\right)-(H f, N f) .
$$

Since $(H f, N f)=0$, and $-\left(\bar{\partial}^{*} \bar{\partial} N f, N f\right)=-\|\bar{\partial} N f\|^{2}$, we see that

$$
\left\|\bar{\partial}^{*} N f\right\|^{2} \leq(f, N f) \leq \epsilon\|f\|^{2}+C_{\epsilon}\|N f\|^{2} .
$$

Lemma 1 now implies that $\bar{\partial}^{*} N$ is compact.

The next proposition is a key step in our proof of Theorem 1. It also could be used instead of Theorem 1 in the proof of Theorem 2 . 
Proposition 1. Suppose that $\Omega$ is a bounded pseudoconvex domain in $\mathbb{C}^{n}$ for which the $\bar{\partial}-N e u m a n n$ operator $N$ is compact on $(0,1)$ forms in $L^{2}(\Omega)$. Let $M$ be a pseudodifferential operator of order 0 . Then the commutator $A=[P, M]$ is compact on $L^{2}(\Omega)$.

Proof. By the formula relating $N$ and $P$ we have

$$
A=[P, M]=\left[I-\bar{\partial}^{*} N \bar{\partial}, M\right]=-\left[\bar{\partial}^{*} N \bar{\partial}, M\right]=-\left[\bar{\partial}^{*}, M\right] N \bar{\partial}+\bar{\partial}^{*}[M, N \bar{\partial}] .
$$

Note first that the commutator of $M$ and either $\bar{\partial}$ or $\bar{\partial}^{*}$ is an operator of order zero, and hence bounded. The first term in the last equality in (6) is the composition of the bounded operator $\left[\bar{\partial}^{*}, M\right]$ with the compact operator $N \bar{\partial}$, and hence is itself compact. The second term is more difficult. We use both lemmas. As usual we write

$$
Q(u, v)=(\bar{\partial} u, \bar{\partial} v)+\left(\bar{\partial}^{*} u, \bar{\partial}^{*} v\right) .
$$

The main property of $Q$ is that $Q(N u, v)=(u, v)$. We write $a=M N \bar{\partial} f-$ $N \bar{\partial} M f$ to simplify notation. Our goal is to show that the map taking $f$ to $\bar{\partial}^{*} a$ is compact. To do so, we compute $Q(a, a)$.

When we commute first order operators past $M$ we obtain operators of order zero, all denoted by $P_{0}$. We obtain

$$
\begin{gathered}
Q(M N \bar{\partial} f-N \bar{\partial} M f, M N \bar{\partial} f-N \bar{\partial} M f)= \\
\|\bar{\partial}(M N \bar{\partial} f-N \bar{\partial} M f)\|^{2}+\left\|\bar{\partial}^{*}(M N \bar{\partial} f-N \bar{\partial} M f)\right\|^{2}= \\
\left(\bar{\partial}^{*} M N \bar{\partial} f, \bar{\partial}^{*} a\right)-\left(\bar{\partial}^{*} N \bar{\partial} M f, \bar{\partial}^{*} a\right)+(\bar{\partial} M N \bar{\partial} f, \bar{\partial} a)-(\bar{\partial} N \bar{\partial} M f, \bar{\partial} a) .
\end{gathered}
$$

Using $Q(N u, v)=(u, v)$, the second and fourth terms in (7) simplify to $-(\bar{\partial} M f, a)$. Using this, commuting $M$ past the differentiations, and moving it to the other side, we obtain

$=\left(P_{0} N \bar{\partial} f, \bar{\partial}^{*} a\right)+\left(\bar{\partial}^{*} N \bar{\partial} f, M^{*} \bar{\partial}^{*} a\right)-(\bar{\partial} M f, a)+\left(P_{0} N \bar{\partial} f, \bar{\partial} a\right)+\left(\bar{\partial} N \bar{\partial} f, M^{*} \bar{\partial} a\right)$

$$
\begin{gathered}
=\left(P_{0} N \bar{\partial} f, \bar{\partial}^{*} a\right)+\left(\bar{\partial}^{*} N \bar{\partial} f, \bar{\partial}^{*} M^{*} a\right)+\left(\bar{\partial}^{*} N \bar{\partial} f, P_{0} a\right)-(\bar{\partial} M f, a) \\
+\left(P_{0} N \bar{\partial} f, \bar{\partial} a\right)+\left(\bar{\partial} N \bar{\partial} f, \bar{\partial} M^{*} a\right)+\left(\bar{\partial} N \bar{\partial} f, P_{0} a\right) .
\end{gathered}
$$

Using $Q(N u, v)=(u, v)$, the second term plus the sixth term in (8) becomes $\left(\bar{\partial} f, M^{*} a\right)$. Subtracting the fourth term gives $([M, \bar{\partial}] f, a)$. Hence we obtain

$$
\begin{aligned}
& Q(a, a)= \\
& \quad\left(P_{0} N \bar{\partial} f, \bar{\partial}^{*} a\right)+\left(P_{0} f, a\right)+\left(\bar{\partial}^{*} N \bar{\partial} f, P_{0} a\right)+\left(P_{0} N \bar{\partial} f, \bar{\partial} a\right)+\left(\bar{\partial} N \bar{\partial} f, P_{0} a\right) \\
& \quad=T_{1}+T_{2}+T_{3}+T_{4}+T_{5} .
\end{aligned}
$$


Our next goal is to show that

$$
Q(a, a) \leq s c\|f\|^{2}+l c\|B f\|^{2},
$$

where $B$ is compact. We estimate each $T_{j}$ :

$$
\begin{gathered}
T_{1} \leq s c\left\|\bar{\partial}^{*} a\right\|^{2}+l c\|N \bar{\partial} f\|^{2} . \\
T_{2} \leq s c\|f\|^{2}+l c\|a\|^{2} .
\end{gathered}
$$

We have

$$
\begin{gathered}
T_{3} \leq s c\left\|\bar{\partial}^{*} N \bar{\partial} f\right\|^{2}+l c\|a\|^{2}=s c\|(I-P) f\|^{2}+l c\|a\|^{2} \leq s c\|f\|^{2}+l c\|a\|^{2} . \\
T_{4} \leq s c\|\bar{\partial} a\|^{2}+l c\|N \bar{\partial} f\|^{2} .
\end{gathered}
$$

We claim that $\bar{\partial} N \bar{\partial} f=0$, so $T_{5}$ vanishes. This follows because the $\bar{\partial}$ and $N$ operators, defined on forms of different degrees, commute. Another proof writes $f=H f+\left(\overline{\partial \partial}^{*}+\bar{\partial}^{*} \bar{\partial}\right) N f$, so $\bar{\partial} f=\overline{\partial \partial}^{*} \bar{\partial} N f$, and thus

$$
\bar{\partial} N \bar{\partial} f=\bar{\partial} N \overline{\partial \partial}^{*} \bar{\partial} N f=\bar{\partial} N\left(\overline{\partial \partial}^{*}+\bar{\partial}^{*} \bar{\partial}\right) \bar{\partial} N f=\overline{\partial \partial} N f=0
$$

Now we can subtract terms of the form $s c\|\bar{\partial} a\|^{2}$ and $s c\left\|\bar{\partial}^{*} a\right\|^{2}$ and obtain an estimate for $Q(a, a)$ involving the other terms. By Lemma $2, N \bar{\partial}$ is compact. Composing it with $M$ on either side still gives a compact operator, because $M$ is bounded. Hence the map sending $f$ to $a=[M, N \bar{\partial}] f$ is compact. Thus we can absorb all the terms on the right in terms of $C\|B f\|^{2}$, where $B$ is compact, except for the $s c\|f\|^{2}$ terms. Since $\left\|\bar{\partial}^{*} a\right\|^{2} \leq Q(a, a)$, we obtain

$$
\left\|\bar{\partial}^{*} a\right\|^{2} \leq Q(a, a) \leq s c\|f\|^{2}+C\|B f\|^{2} .
$$

Statement (10) and Lemma 1 imply the desired conclusion.

We will use Theorem 1 in order to prove Theorem 2; a weaker version (for polynomials vanishing on the diagonal) suffices for our application. If we assume that $g$ is a polynomial vanishing on the diagonal, then we can prove Theorem 1 assuming only that $N$ is compact by using Proposition 1 .

Theorem 1. Suppose that $\Omega$ is a smoothly bounded pseudoconvex domain of finite type with Bergman kernel function B. Suppose that $g$ is smooth on $\bar{\Omega} \times \bar{\Omega}$ and that $g$ vanishes on the boundary diagonal. Let $T: L^{2}(\Omega) \rightarrow L^{2}(\Omega)$ be the operator whose kernel is given by $g B$. Then $T$ is a compact operator.

Proof. We will write $g(z, \zeta)$ for the value of $g$; this notation does not mean that $g$ is holomorphic in either variable! First we write $z=\zeta+(z-\zeta)$. We can therefore write by Taylor's formula

$$
g(z, \zeta)=g(\zeta+(z-\zeta), \zeta)=g_{0}(\zeta)+\sum_{|\alpha|,|\beta| \leq N} f_{\alpha \beta}(\zeta)(z-\zeta)^{\alpha}(\bar{z}-\bar{\zeta})^{\beta}+R_{N}
$$


Here $R_{N}$ is the remainder term. Evaluating (11) at $z=\zeta$ gives $g(\zeta, \zeta)=g_{0}(\zeta)$. Since $g$ vanishes on the boundary diagonal we conclude that $g_{0}$ vanishes on the boundary.

Each of these terms gets multiplied by the Bergman kernel. We first show that $g_{0}(\zeta) B(z, \bar{\zeta})$ defines a compact operator $\mathcal{A}$. To establish the compactness, we use Lemma 1. Suppose that $r$ is a defining function for $\Omega$. Given $\epsilon>0$, we can find $\delta>0$ so that $-\delta \leq r \leq 0$ implies $\left|g_{0}\right| \leq \epsilon$. Choose a smooth positive function $\chi$, bounded by unity, that equals unity when $r<-\delta$, and is supported in $\Omega$. Then we write

$$
g_{0} B=\chi g_{0} B+(1-\chi) g_{0} B=A_{1}+A_{2} .
$$

The operator $\mathcal{A}_{1}$ defined by $A_{1}$ is compact, since its kernel is smooth on all of $\bar{\Omega} \times \bar{\Omega}$. The operator $\mathcal{A}_{2}$ defined by $A_{2}$ is multiplication by $g_{0}(1-\chi)$ followed by the Bergman projection $P$. Note that $\left|g_{0}\right| \leq \epsilon$ where $\chi$ is not equal to one. Hence we can write

$$
\begin{aligned}
\|\mathcal{A} f\|=\left\|\mathcal{A}_{1} f+\mathcal{A}_{2} f\right\| & \leq\left\|\mathcal{A}_{1} f\right\|+\left\|P(1-\chi) g_{0} f\right\| \\
& \leq\left\|\mathcal{A}_{1} f\right\|+\left\|(1-\chi) g_{0} f\right\| \leq\left\|\mathcal{A}_{1} f\right\|+\epsilon\|f\| .
\end{aligned}
$$

Thus $\mathcal{A}$ defines a compact operator by Lemma 1 .

We now consider the sum in (11). Each of the terms $f_{\alpha \beta}(\zeta)(z-\zeta)^{\alpha}(\bar{z}-\bar{\zeta})^{\beta}$ is a finite sum of terms in the form $A_{j}(z) B_{j}(\zeta)\left(z_{j}-\zeta_{j}\right)$ or $A_{j}(z) B_{j}(\zeta)\left(\bar{z}_{j}-\bar{\zeta}_{j}\right)$ where $A_{j}$ and $B_{j}$ are smooth. The composition in either order of a compact operator with a bounded operator is a compact operator, and a finite sum of compact operators is a compact operator. Therefore, to establish the compactness of $A$, it suffices to prove the following: Suppose that $A: L^{2} \rightarrow L^{2}$ is the operator whose kernel is given by $\left(\bar{\zeta}_{j}-\bar{z}_{j}\right) B(z, \bar{\zeta})$, or by $\left(z_{j}-\zeta_{j}\right) B(z, \bar{\zeta})$. Then $A$ is compact.

Thus the crucial point is to show that such an $A$ is compact. Note however that such an $A$ is the commutator $[P, M]$, where $M$ denotes multiplication by $\bar{z}_{j}$ or its conjugate. Thus the compactness of $A$ follows from Proposition 1.

Finally we must handle the remainder term from (11). It suffices to show that the kernel $R_{N}(z, \zeta) B(z, \bar{\zeta})$ is continuous on $\bar{\Omega} \times \bar{\Omega}$. To see this it suffices to show that there is a positive exponent $\alpha$ such that

$$
|B(z, \bar{\zeta})| \leq C|z-\zeta|^{-\alpha}
$$

If (12) holds, then we can choose the index $N$ in the Taylor expansion (11) so large that $R_{N}(z, \zeta) B(z, \bar{\zeta})$ is continuous on $\bar{\Omega} \times \bar{\Omega}$, and therefore defines a compact operator. We state and prove (12) in Lemma 3 ; its proof completes the proof of Theorem 1.

Lemma 3. Suppose that $\Omega$ is a smoothly bounded pseudoconvex domain for which a subelliptic estimate of order $\epsilon$ holds on $(0,1)$ forms. Then there is a constant $C$ so that

$$
|B(z, \bar{\zeta})| \leq C|z-\zeta|^{-(2 n+6) / \epsilon}
$$


Proof. The proof follows the methods of $[\mathrm{Ke}]$ and [C2]. First we assume that $z \in \bar{\Omega}$, that $\zeta \in \Omega$, and that the distance between them is $t$. Choose $\delta$ equal to the minimum of $\frac{t}{4}$ and the distance between $\zeta$ and the boundary. Let $\phi_{\zeta}$ be an approximation to the identity at $\zeta$, spherically symmetric and supported in a ball about radius $\delta$ about $\zeta$. We may write

$$
B(z, \zeta)=\left(I-\bar{\partial}^{*} N \bar{\partial}\right)\left(\phi_{\zeta}\right)(z)
$$

Choose smooth cut-off functions $\chi_{1}$ and $\chi_{2}$ supported in a ball of radius $\frac{t}{4}$ and so that $\chi_{2}=1$ near $\operatorname{supp}\left(\chi_{1}\right)$. Using the methods of Proposition 2 from $[\mathrm{C} 2]$, there is an estimate involving Sobolev norms:

$$
\left|\chi_{1} B(., \zeta)\right| \leq\left\|\chi_{1} \bar{\partial}^{*} N \bar{\partial} \phi_{\zeta}\right\|_{n+1} \leq C t^{-\frac{n+3}{\epsilon}}\left\|\chi_{2} N \bar{\partial} \phi_{\zeta}\right\|
$$

We estimate $\left\|\chi_{2} N \bar{\partial} \phi_{\zeta}\right\|$ by pairing $\chi_{2} N \bar{\partial} \phi_{\zeta}$ with a smooth $(0,1)$ form $f$ with $\|f\|_{0} \leq 1$. We obtain using integration by parts that

$$
\left|\left(\chi_{2} N \bar{\partial} \phi_{\zeta}, f\right)\right|=\left|\left(\phi_{\zeta}, \bar{\partial}^{*} N \chi_{2} f\right)\right| \leq\left|\left(\phi_{\zeta}, \lambda \bar{\partial}^{*} N \chi_{2} f\right)\right|
$$

where we have written $\lambda$ for a cut-off function that is unity near $\operatorname{supp}\left(\phi_{\zeta}\right)$, vanishes near $\operatorname{supp}\left(\chi_{2}\right)$, and whose $k$-th derivative can be estimated by $C_{k} t^{-k}$. Using the generalized Schwartz inequality we can estimate (15) by

$$
(15) \leq\left\|\phi_{\zeta}\right\|_{-n-1}\left\|\lambda \bar{\partial}^{*} N \chi_{2} f\right\|_{n+1} \leq C\left\|\phi_{\zeta}\right\|_{-n-1} t^{-\frac{n+3}{\epsilon}}\|f\| .
$$

Taking the supremum over $f$ and estimating $\left\|\phi_{\zeta}\right\|_{-n-1}$ by a positive constant, we obtain

$$
|B(z, \zeta)| \leq C t^{-(2 n+6) / \epsilon},
$$

which gives (13). Finally, since $B$ is smooth on $\bar{\Omega} \times \bar{\Omega}$ off the diagonal, the same estimate holds when $z, \zeta \in \bar{\Omega}$.

\section{Proof of the main result}

In this section we write $\|z\|^{2}$ for the Euclidean norm of a point in $\mathbb{C}^{n}$. Let $\Omega$ be a bounded domain in $\mathbb{C}^{n}$. We now write $\langle,\rangle_{\Omega}$ for the $L^{2}$ inner product given by

$$
\langle g, h\rangle_{\Omega}=\int_{\Omega} g(z) \bar{h}(z) d V(z)
$$

and write $\|g\|_{\Omega}^{2}$ for $\langle g, g\rangle_{\Omega}$. Recall that $\Omega$ is circled if it is invariant under scalar multiplication by $e^{i \theta}$. We have the following simple lemma. 
Lemma 4. Suppose that $\Omega$ is a bounded circled domain. Then, for $j \neq k$, the spaces $V_{j}$ and $V_{k}$ of holomorphic homogeneous polynomials are orthogonal in $A^{2}(\Omega)$.

Proof. Suppose that $p_{j}$ and $p_{k}$ are homogeneous polynomials of the degree indicated. Then we have $p_{j}\left(e^{i \theta} z\right)=e^{i j \theta} p_{j}(z)$. From the definition of the inner product as an integral, the change of variables formula for integrals, and the invariance of $\Omega$ under multiplication by $e^{i \theta}$, we obtain

$$
\begin{aligned}
\left\langle p_{j}, p_{k}\right\rangle_{\Omega} & =\int_{\Omega} p_{j}(z) \bar{p}_{k}(z) d V(z) \\
& =\int_{\Omega} p_{j}\left(e^{i \theta z}\right) \bar{p}_{k}\left(e^{i \theta z}\right) d V\left(e^{i \theta z}\right)=e^{i \theta(j-k)}\left\langle p_{j}, p_{k}\right\rangle_{\Omega} .
\end{aligned}
$$

From (17) we see that the inner product must vanish unless $j=k$.

Let $N=N(d, n+1)$ denote the dimension of $V_{d}$. Suppose that $\left(E_{\mu \nu}\right)$ is a Hermitian matrix on $\mathbb{C}^{N}$. We consider the integral operator $\mathcal{E}$ defined on $V_{d}$ by

$$
(\mathcal{E} g)(z)=\int_{\Omega} \sum_{\mu, \nu} E_{\mu \nu} z^{\mu} \bar{w}^{\nu} g(w) d V(w) .
$$

Proposition 2. Let $\Omega$ be a bounded circled domain. The following are equivalent:

1. The Hermitian matrix $\left(E_{\mu \nu}\right)$ is positive definite.

2. The operator $\mathcal{E}$ defined by (18) is positive definite.

3. We can write

$$
f(z, \bar{z})=\sum E_{\mu \nu} z^{\mu} \bar{z}^{\nu}=\|A(z)\|^{2}
$$

where the components of $A$ form a basis for $V_{d}$.

Proof. Suppose that 1) holds. By linear algebra we can find basis vectors $E_{\mu}$ of $\mathbb{C}^{N}$ so that $E_{\mu \nu}=\left\langle E_{\mu}, E_{\nu}\right\rangle$. This implies that $f(z, \bar{z})=\left\|\sum E_{\mu} z^{\mu}\right\|^{2}$, so 1$)$ implies 3).

If 3) holds we have $f(z, \bar{z})=\|A(z)\|^{2}$, where $A_{1}, A_{2}, \ldots, A_{N}$ form a basis for $V_{d}$. Then

$$
\langle\mathcal{E} h, h\rangle_{\Omega}=\int_{\Omega} \int_{\Omega} \sum A_{j}(z) \overline{A_{j}(\zeta)} h(\zeta) \overline{h(z)} d V(\zeta) d V(z)=\sum\left|\left\langle A_{j}, h\right\rangle_{\Omega}\right|^{2}
$$

and we see that $\mathcal{E}$ is a positive operator. Thus 3 ) implies 2).

We finish the proof by showing that 2) implies 1). Thus we want to find $k>0$ so that

$$
\sum E_{\mu \nu} \zeta_{\mu} \bar{\zeta}_{\nu} \geq k \sum\left|\zeta_{\mu}\right|^{2}
$$


Define $c_{\alpha \beta}=\left\langle z^{\alpha}, z^{\beta}\right\rangle_{\Omega}$. Since this matrix is invertible we may define $g_{\beta}$ by $\sum c_{\beta \nu} g_{\beta}=\zeta_{\nu}$. Let $g=\sum g_{\alpha} z^{\alpha}$. Because $\mathcal{E}$ is positive definite, there is a $c>0$ so that

$$
\langle\mathcal{E} g, g\rangle_{\Omega} \geq c\|g\|_{\Omega}^{2}
$$

Doing simple computations shows that

$$
\begin{gathered}
\langle\mathcal{E} g, g\rangle_{\Omega}=\sum E_{\mu \nu} c_{\mu \alpha} c_{\beta \nu} \bar{g}_{\alpha} g_{\beta} \\
\|g\|_{\Omega}^{2}=\sum c_{\alpha \beta} g_{\alpha} \bar{g}_{\beta} .
\end{gathered}
$$

The matrix $\left(c_{\alpha \beta}\right)$ arises from inner products and hence is positive definite. Hence there are constants so that $\|g\|_{\Omega}^{2} \geq c^{\prime} \sum\left|g_{\alpha}\right|^{2} \geq c^{\prime \prime} \sum\left|\zeta_{\alpha}\right|^{2}$. Therefore we have

$$
\sum E_{\mu \nu} \zeta_{\mu} \bar{\zeta}_{\nu}=\sum E_{\mu \nu} c_{\mu \alpha} c_{\beta \nu} \bar{g}_{\alpha} g_{\beta}=\langle\mathcal{E} g, g\rangle_{\Omega} \geq c\|g\|_{\Omega}^{2} \geq c c^{\prime \prime} \sum\left|\zeta_{j}\right|^{2}
$$

This shows that 2) implies 1).

We can now state and prove our main application of Theorem 1.

Theorem 2. Suppose that $\Omega$ is a smoothly bounded circled pseudoconvex domain of finite type in $\mathbb{C}^{n}$. For each integer $d \geq 0$, let $\Phi^{d}=\left(\Phi_{1}^{d}, \ldots, \Phi_{N}^{d}\right)$ denote an orthonormal basis for the homogeneous polynomials of degree $d$ on $\Omega$. Let $f$ be a bihomogeneous polynomial that is positive away from the origin. Then there is an integer $d_{0}$ such that, for each $d \geq d_{0}$, there is a homogeneous polynomial mapping $h$ such that

$$
\left\|\Phi^{d}(z)\right\|^{2} f(z, \bar{z})=\|h(z)\|^{2} .
$$

Proof. We will prove that, for all sufficiently large $d$, there is a homogenoeus polynomial mapping $h$ such that (21) holds. To do this we use Proposition 2; (21) holds if and only if the operator $K$ with integral kernel

$$
f(z, \bar{\zeta})\left\langle\Phi^{d}(z), \Phi^{d}(\zeta)\right\rangle
$$

is positive on the space $V_{m+d}$.

To prove this, we first let $\xi$ be a smooth function with compact support in $\Omega$ that is positive at the origin. We write

$f(z, \bar{\zeta}) B(z, \bar{\zeta})=(f(z, \bar{z})+\xi(\zeta, \bar{\zeta})) B(z, \bar{\zeta})-\xi(\zeta, \bar{\zeta}) B(z, \bar{\zeta})+(f(z, \bar{\zeta})-f(z, \bar{z})) B(z, \bar{\zeta})$

$$
=T_{1}+T_{2}+T_{3}
$$

We claim that the first term $T_{1}$ defines a positive operator $Q$ on all of $A^{2}(\Omega)$, and that the operators defined by $T_{2}$ and $T_{3}$ are compact. The first follows because 
the Bergman kernel is a self-adjoint projection. To use this, let $h$ be in $A^{2}(\Omega)$. Then $Q h=M_{f} P h+P M_{\xi} h=M_{f} h+P M_{\xi} h$. Here $M_{q}$ is the operator given by multiplication by $q$. Therefore we have

$$
\begin{aligned}
\langle Q h, h\rangle_{\Omega} & =\left\langle M_{f} h+P M_{\xi} h, h\right\rangle_{\Omega} \\
& =\left\langle M_{f} h, h\right\rangle_{\Omega}+\left\langle M_{\xi} h, P h\right\rangle=\left\langle M_{f+\xi} h, h\right\rangle_{\Omega} \geq c\|h\|_{\Omega}^{2} .
\end{aligned}
$$

In $(23)$ we have estimated $f+\xi$ from below by a positive constant. This proves that $Q$ is positive.

The operator defined by $T_{2}$ is compact because $T_{2}$ is smooth on all of $\bar{\Omega} \times \bar{\Omega}$. Since $B$ is holomorphic in its first variable, and anti-holomorphic in its second variables, it is smooth on $\Omega \times \Omega$. Since $\xi$ has compact support, $T_{2}$ is smooth everywhere. Given our hypotheses on $\Omega$, Theorem 1 implies that the operator defined by $T_{3}$ is also compact.

Observe that $K$ is now known to be the sum of a compact operator $T$ and an operator $Q$ that it positive on $A^{2}(\Omega)$. Suppose that $\langle Q h, h\rangle_{\Omega} \geq c\|h\|_{\Omega}^{2}$ for all $h \in A^{2}(\Omega)$. Write $\|T\| \|$ for the operator norm of $T$. Since $T$ is compact, we can find $[\mathrm{Ru}]$ a finite rank operator $L$ so that $\left\||T-L \||<\frac{c}{3}\right.$. Since $L$ is finite rank, for sufficiently large $j$ we may assume that the restriction $L^{\prime}$ of $L$ to $V_{j}$ has operator norm ||$L^{\prime}|| \mid<\frac{c}{3}$. Therefore, on $V_{j}$, for sufficiently large $j$

$$
\begin{aligned}
\langle K h, h\rangle_{\Omega} & =\langle Q h, h\rangle_{\Omega}+\langle(T-L) h, h\rangle_{\Omega}+\langle L h, h\rangle_{\Omega} \\
& \geq c\|h\|_{\Omega}^{2}-\frac{c}{3}\|h\|_{\Omega}^{2}-\frac{c}{3}\|h\|_{\Omega}^{2}=\frac{c}{3}\|h\|_{\Omega}^{2} .
\end{aligned}
$$

By (24) $K$ is positive on $V_{j}$. Choose a complete orthonormal set for $A^{2}(\Omega)$ including all components of $\Phi_{d}$, and replace $B(z, \bar{\zeta})$ by the orthonormal series $\sum \phi_{\alpha}(z) \overline{\phi_{\alpha}(\zeta)}$. The restriction of $K$ to $V_{j}$ is then given by the kernel $f(z, \bar{\zeta})\left\langle\Phi^{d}(z), \Phi^{d}(\zeta)\right\rangle$. By the positivity and by Proposition 2, it follows that $f(z, \bar{z})\left\|\Phi^{d}(z)\right\|^{2}$ is a squared norm as in (21).

\section{Interpretation in terms of line bundles}

Let $\mathbb{P}_{n}$ denote the complex projective space of lines through the origin in $\mathbb{C}^{n+1}$. As usual, see $[\mathrm{W}]$ for example, for $j=0,1, \ldots, n$ we let $U_{j}$ be the open subset where the homogeneous coordinate $z_{j}$ is not zero. A line bundle $E$ over $\mathbb{P}_{n}$ is defined by its transition functions $g_{j k}: U_{j} \cap U_{k} \rightarrow \mathbb{C}^{*}$. Recall that the universal line bundle $\mathbb{U}$ over $\mathbb{P}_{n}$ is the line bundle whose transition functions are $h_{j k}(z)=\frac{z_{j}}{z_{k}}$. The hyperplane section bundle $H$ over $\mathbb{P}_{n}$ is the dual line bundle; its transition functions are $g_{j k}(z)=\frac{z_{k}}{z_{j}}$. The $m$-th powers of these bundles are the bundles $\mathbb{U}^{m}$ and $H^{m}$ whose transition functions are $\left(\frac{z_{j}}{z_{k}}\right)^{m}$ and $\left(\frac{z_{k}}{z_{j}}\right)^{m}$ respectively. The holomorphic sections of $H^{m}$ are homogeneous polynomials of degree $m$ in the homogeneous coordinates.

A metric on a line bundle $E$ over $\mathbb{P}_{n}$ whose transition functions are $g_{j k}$ determines a positive function $p_{j}$ in each $U_{j}$ such that $p_{j}=p_{k}\left|g_{k j}\right|^{2}$ on the overlap 
$U_{j} \cap U_{k}$, and conversely such a collection of positive functions defines a metric on $E$. This is easily seen by observing that if $s_{k}$ denotes the representation of a local section in $U_{k}$, then its squared length $p_{k}\left|s_{k}\right|^{2}$ must equal $p_{j}\left|s_{j}\right|^{2}$ in the overlap $U_{j} \cap U_{k}$. Since $s_{k}=g_{k j} s_{j}$ we see that the metric must transform by

$$
p_{j}=p_{k}\left|g_{k j}\right|^{2} .
$$

Let $\mathcal{P}$ denote the set of bihomogeneous polynomials on $\mathbb{C}^{n+1}$ that are positive away from the origin. When $f \in \mathcal{P}$, we can use it to define metrics on $\mathbb{U}^{m}$ or on $H^{m}$ over $\mathbb{P}_{n}$ by the following method. For $j=0, \ldots, n$ we put

$$
f_{j}(z, \bar{z})=\frac{f(z, \bar{z})}{\left|z_{j}\right|^{2 m}}
$$

Of course $f_{j}$ is defined and positive in $U_{j}$. On $U_{j} \cap U_{k}$, we have

$$
\frac{f_{j}}{f_{k}}=\left|\left(\frac{z_{k}}{z_{j}}\right)^{m}\right|^{2}
$$

Therefore the collection of functions $f_{j}$ defines a metric on the line bundle whose transition functions are $h_{j k}=\left(\frac{z_{k}}{z_{j}}\right)^{m}$. This is the bundle $\mathbb{U}^{m}$.

Had we put

$$
p_{j}(z, \bar{z})=\frac{\left|z_{j}\right|^{2 m}}{f(z, \bar{z})}
$$

then the functions $p_{j}$ would define a metric on the $m$-th power of the hyperplane section bundle $H^{m}$.

Definition 1. We say that a metric defined on $\mathbb{U}^{m}$ or $H^{m}$ is a special metric if it is defined by a bihomogeneous positive polynomial $f$ as in $(\mathrm{U})$ or $(\mathrm{H})$ above. We write the bundle and the special metric on $\mathbb{U}^{m}$ as the ordered pair $\left(\mathbb{U}^{m}, f\right)$.

Next we consider the effect of multiplying $f$ by the squared norm $\left\|\Phi^{d}\right\|^{2}$ in Theorem 2. Since $\left\|\Phi^{d}\right\|^{2} \in \mathcal{P}$, it defines a metric on $\mathbb{U}^{d}$ or $H^{d}$ as above.

In the case of the unit ball, we use $\|z\|^{2}$ to define a special metric on $\mathbb{U}$; this is the standard metric. When we raise the squared norm to the power $d$, we obtain metrics on $\mathbb{U}^{d}$ or $H^{d}$. This amounts to taking the $d$-fold tensor product of the bundle. The resulting metric differs only by constants from the metrics defined by $\left\|\Phi^{d}\right\|^{2}$.

We may consider $\|z\|^{2 d} f(z, \bar{z})$ as a metric on $\mathbb{U}^{m+d}$ or $H^{m+d}$ in the same way. Using tensor products we can write

$$
\left(\mathbb{U}^{d},\|z\|^{2 d}\right) \otimes\left(\mathbb{U}^{m}, f(z, \bar{z})\right)=\left(\mathbb{U}^{m+d},\|z\|^{2 d} f(z, \bar{z})\right) .
$$

Suppose that $\|z\|^{2 d} f(z, \bar{z})=\|g\|^{2}$ and the components of $g$ form a basis for $V_{m+d}$. Let $N=N(m+d, n+1)$ denote the dimension of the vector space 
of homogeneous polynomials of degree $m+d$ in $n+1$ complex variables, and consider the universal bundle $\mathbb{U}$ over $\mathbb{P}_{N}$. The line bundle $\mathbb{U}^{m+d}$ over $\mathbb{P}_{n}$ is obviously the pullback by $g$ of the line bundle $\mathbb{U}$ over $\mathbb{P}_{N}$. The metric is also given by a pullback. If we equip $\mathbb{U}$ with the metric given by $\|L(\zeta)\|^{2}$, where $L$ is the appropriate invertible linear mapping, then $\left(\mathbb{U}^{m+d},\|g(z)\|^{2}\right)$ over $\mathbb{P}_{n}$ is the pullback of $\left(\mathbb{U},\|L(\zeta)\|^{2}\right)$ over $\mathbb{P}_{N}$.

We can now restate Theorem [CD] and also Theorem 2.

Theorem 3. Let $\left(\mathbb{U}^{m}, f\right)$ denote the $m$-th power of the universal line bundle over $\mathbb{P}_{n}$ with special metric defined by $f$. Then there is an integer $d$ so that $\left(\mathbb{U}^{m+d},\|z\|^{2 d} f(z, \bar{z})\right)$ is a (holomorphic) pullback $g^{*}\left(\mathbb{U},\|L(\zeta)\|^{2}\right)$ of the standard metric on the universal bundle over $\mathbb{P}_{N}$. The mapping $g: \mathbb{P}_{n} \rightarrow \mathbb{P}_{N}$ is a holomorphic (polynomial) embedding and $L$ is an invertible linear mapping.

$$
\left(\mathbb{U}^{m}, f\right) \otimes\left(\mathbb{U}^{d},\|z\|^{2 d}\right)=\left(\mathbb{U}^{m+d},\|z\|^{2 d} f(z, \bar{z})\right)=\left(\mathbb{U}^{m+d},\|g(z)\|^{2}\right) .
$$

We have the bundles and metrics

$$
\begin{gathered}
\pi_{1}:\left(\mathbb{U}^{m}, f\right) \rightarrow \mathbb{P}_{n} \\
\pi_{2}:\left(\mathbb{U}^{m+d},\|z\|^{2 d} f\right) \rightarrow \mathbb{P}_{n} \\
\pi_{3}:\left(\mathbb{U},\|L(\zeta)\|^{2}\right) \rightarrow \mathbb{P}_{N} .
\end{gathered}
$$

The first is not necessarily a pullback of the third, but for sufficiently large $d$, the second must be. We conclude the paper by restating Theorem 2 in this language.

Theorem 4. Suppose that $\Omega$ is a smoothly bounded pseudoconvex circled domain of finite type in $\mathbb{C}^{n}$. Let $\left(\mathbb{U}^{d},\left\|\Phi^{d}\right\|^{2}\right)$ denote the $d$-th power of the universal bundle over $\mathbb{P}_{n-1}$ with special metric $\left\|\Phi^{d}\right\|^{2}$. Here

$$
\left\|\Phi^{d}\right\|^{2}=\sum \phi_{\alpha}(z) \overline{\phi_{\alpha}(z)}
$$

and the sum is taken over an orthonormal basis for $V_{d}$. Let $f$ define a special metric on $\left(\mathbb{U}^{m}, f\right)$. Then there is an integer $d_{0}$ such that, for all $d \geq d_{0}$, the bundle $\left(\mathbb{U}^{m+d}, f\left\|\Phi^{d}\right\|^{2}\right)$ is the holomorphic pullback of the universal line bundle $\left(\mathbb{U},\|L(\zeta)\|^{2}\right)$ over some $\mathbb{P}_{N}$ with the standard metric. Again $L$ is an invertible linear mapping.

\section{References}

[C1] D. Catlin, Subelliptic estimates for the $\bar{\partial}-$ Neumann problem on pseudoconvex domains, Ann. of Math. 126 (1987), 131-191.

[C2] Necessary conditions for subellipticity of the $\bar{\partial}$-Neumann problem, Ann. of Math. 117 (1983), 147-171. 
[CD] D. Catlin and J. P. D'Angelo, A stabilization theorem for Hermitian forms and applications to holomorphic mappings, Math Res. Lett. 3 (1996), 149-166.

[D] J. P. D'Angelo, Several complex variables and the geometry of real hypersurfaces (1993), CRC Press, Boca Raton.

[De] J.-P. Demailly, $L^{2}$ vanishing theorems for positive line bundles and adjunction theory, CIME Session, Transcendental Methods in Algebraic Geometry, Cetraro Italy, (1994).

[FK] G. B. Folland and J. J. Kohn, The Neumann problem for the Cauchy-Riemann complex, Ann. of Math Studies, 75, Princeton University Press, 1972.

[HI] G. M. Henkin and A. Iordan, Compactness of the Neumann operator for hyperconvex domains with non-smooth boundaries, Math. Ann. 307 (1997), 151-169.

[Ke] N. Kerzman, The Bergman kernel: Differentiability at the boundary, Math Annalen 195 (1972), 149-158.

[K] J. J. Kohn, Subellipticity of the $\bar{\partial}-$ Neumann problem on pseudoconvex domains: Sufficient conditions, Acta Math 142 (1979), 79-122.

[Ru] W. Rudin, Functional analysis (1973), McGraw-Hill, New York.

[W] R. O. Wells, Differential analysis on complex manifolds (1973), Prentice-Hall, Englewood Cliffs, New Jersey.

Dept. of Mathematics, Purdue Univ., W. Lafayette IN 47907

E-mail address: catlin@math.purdue.edu

Dept. of Mathematics, Univ. of Illinois, Urbana IL 61801

E-mail address: jpda@math.uiuc.edu 